\title{
The Mathematical Sciences Center of Tsinghua University
}

\author{
Yat Sun Poon
}

Department of Mathematics, University of California at Riverside

\section{Founding of a Center}

In its one hundred years, Tsinghua has long been known as a leading university in China. It is internationally well known for its extremely strong engineering and applied science programs. Tsinghua University also recognizes the fundamental and strategic role mathematics play in its science program. In December 2009, Tsinghua University inaugurated the Mathematical Sciences Center (MSC), and invited Professor Shing-Tung Yau to serve as its inaugural director. This invitation came with very ambitious commitment and expectation from the core leadership of the Tsinghua University. The commitment provides with time and opportunity for the Center to develop to its full potential.

As noted by Yau: "The goal of establishing the MSC is to push the development of mathematical sciences in Tsinghua and in China to international frontier. It will serve as a forum for the accomplished mathematicians to

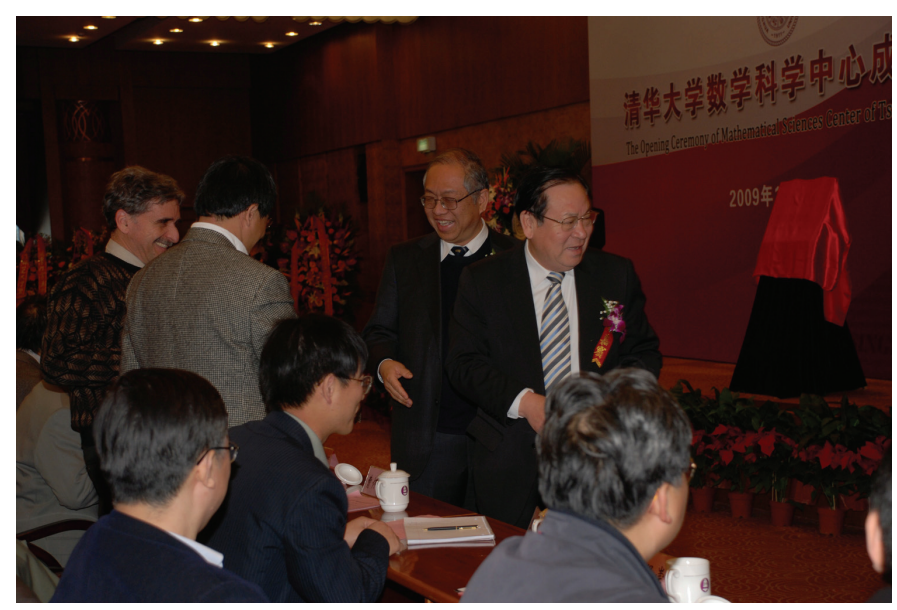

2009 Inauguration Ceremony

nurture a new generation of scientific leaders. It will be an incubator for early-career mathematicians to develop their most original and creative works. It will be a lively classroom for students to learn a good spectrum of mathematics, from foundational to the most advanced." To put his words in action, Yau started a forceful recruitment drive as soon as fall 2009, and has been working tirelessly on it ever since. As one of his close advisors on MSC affairs, I witness the growth of the MSC through its recruitment of full-time or long-term members, establishment of visiting professors program, support due to a large international community, development

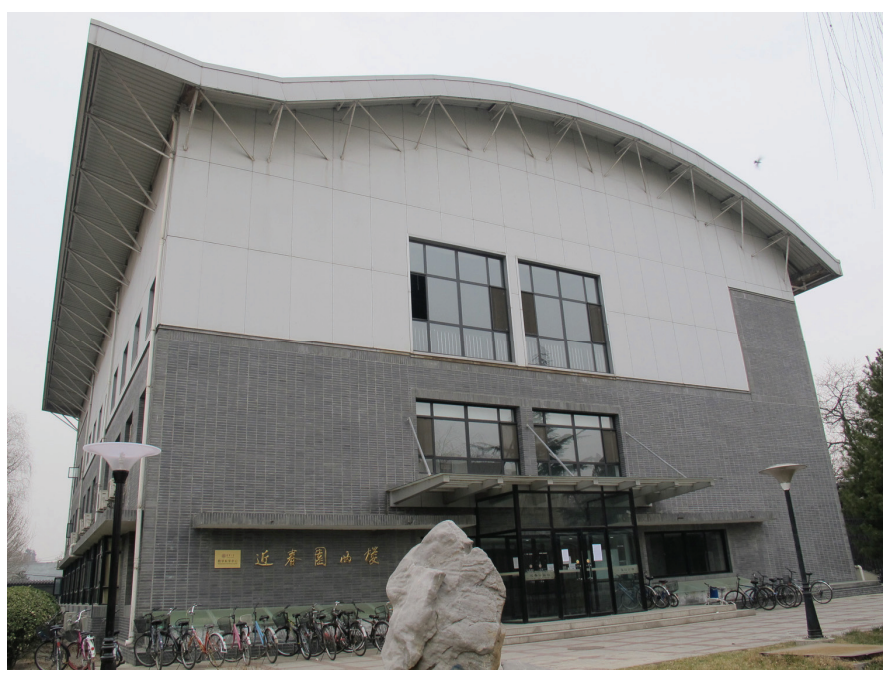

The Mathematical Sciences Center of Tsinghua University

of academic programs, recruitment of graduate students, cultivation of talents, and its capital campaign.

In its recruitment campaign, the MSC not only focuses on recruiting leaders or young talents to its team, but also requires its members to make a sufficient contribution to the research and teaching environment in Tsinghua. This requirement secures significant attention of all members on the academic well-being of the Center and all junior members associated to it. It sustains interaction between members of the Center and the local students as well as all members of the mathematics community in Tsinghua.

By December 2012, the MSC had secured the following full-time memberships: 
- Ben Andrews (1993 PhD. Australian National University) Geometric Analysis

- Ning Jiang (2006 PhD. Maryland) Nonlinear Partial Differential Equations

- Tao Luo (1995 PhD. Chinese Academy of Sciences) Nonlinear Partial Differential equations

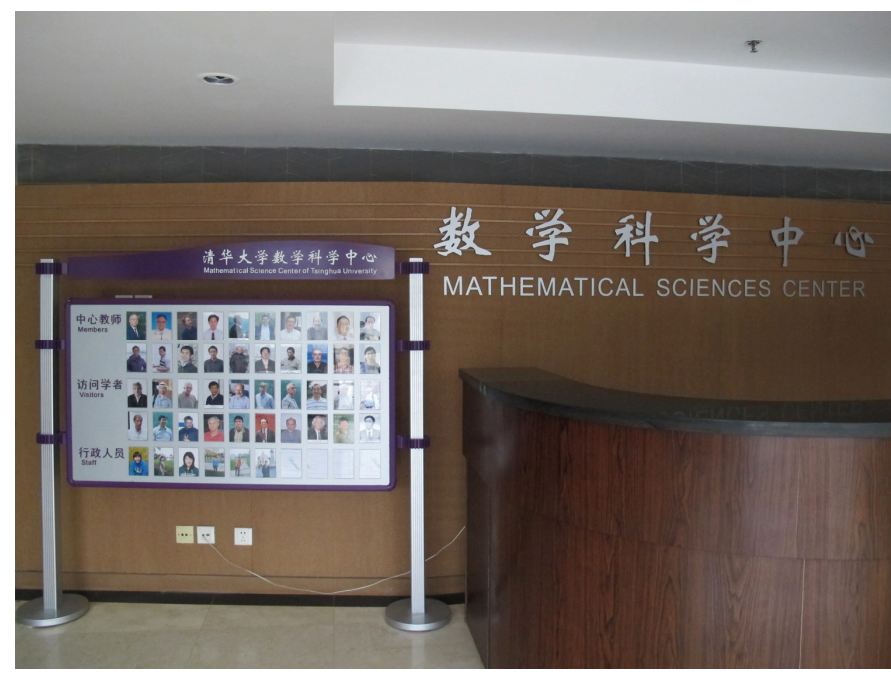

The Mathematical Sciences Center of Tsinghua University

- Abul Masood-ul-Alam (1986 PhD. Australian National University) General Relativity

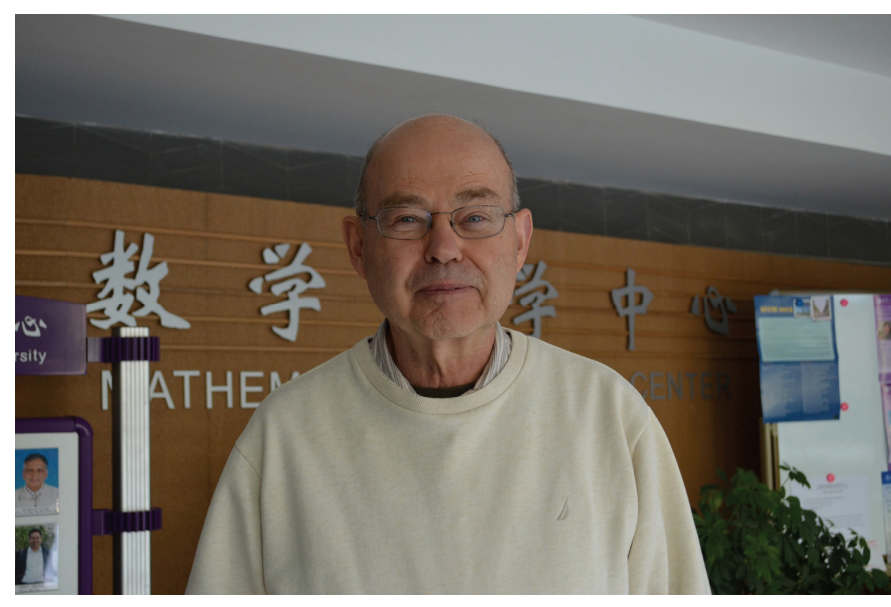

Thomas Farrell

- Zuoqiang Shi (2008 PhD. Tsinghua) Data Analysis and Fluid Structure

- Jian Sun (2007 PhD. Ohio State) Imaging, Computing and Geometry

- Shenghao Sun (2010 PhD. Berkeley) Algebraic Geometry
- $\quad$ Pin Yu (2010 PhD. Princeton) Geometric Analysis

- Kai Fun Yu (1978 PhD. Columbia) Statistics

- Huihui Zeng (2009 PhD. The Chinese University of Hong Kong) Nonlinear Partial Differential Equations

- Yuguang Zhang (2006 PhD. Nankai) Geometric Analysis

- Minxian Zhu (2008 PhD. Yale) Algebra

In the addition, the following mathematicians are part-time, long-term members of the MSC:

- Spencer Bloch (1971 PhD. Columbia) University of Chicago, Algebraic Geometry

- Yat Sun Poon (1986 D.Phil. Oxford) University of California, Riverside, Differential Geometry

- Tonghai Yang (1995 PhD. Maryland) University of Wisconsin, Algebraic Geometry

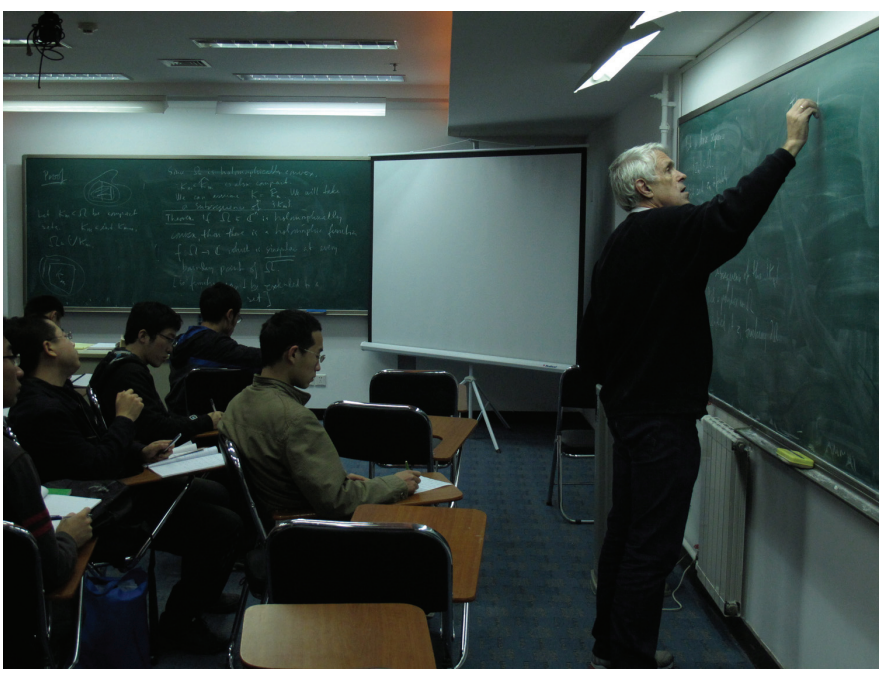

John Erik Fornaess

- Jun Liu (1991 PhD. Chicago) Harvard University, Statistics

By Fall 2013 the following group of mathematicians will join the MSC full time:

- Shiu Yuen Cheng (1974 PhD. Berkeley) Differential Geometry

- Thomas Farrell (1967 PhD. Yale) Beginning in fall 2014, Topology

- Eduard Looijenga (1974 PhD. Amsterdam) Algebraic Geometry

- John Erik Fornaess (1974 PhD. Washington) Complex Analysis

- Ke Deng (2008 PhD. Peking) Statistics

- Hiroyuki Fuji (2002 PhD. Tokyo) String Theory

- Wenxuan Lu (2011 PhD. MIT) String Theory 
- François-Xavier Machu (2008 PhD. Lille) Algebraic Geometry

- Guoyi Xu (2010 PhD. Minnesota) Geometric Analysis In addition, the Center works in close collaboration with the Department of Mathematical Sciences of Tsinghua University. Beginning in spring 2012, the collaboration is formalized by the adjunct appointment of six departmental faculty members to be MSC members. They are Professors Ning Su (Partial Differential Equations), Haizhong Li (Global Differential Geometry), Jiaxin Hu (Partial Differential Equation and Fractal Geometry), Guangyuan Zhang (Complex Analysis), Li Chen (Partial Differential Equations), and Fan Xu (Representation Theory).

\section{Visiting Professor Program and Distinguished Lecture Series}

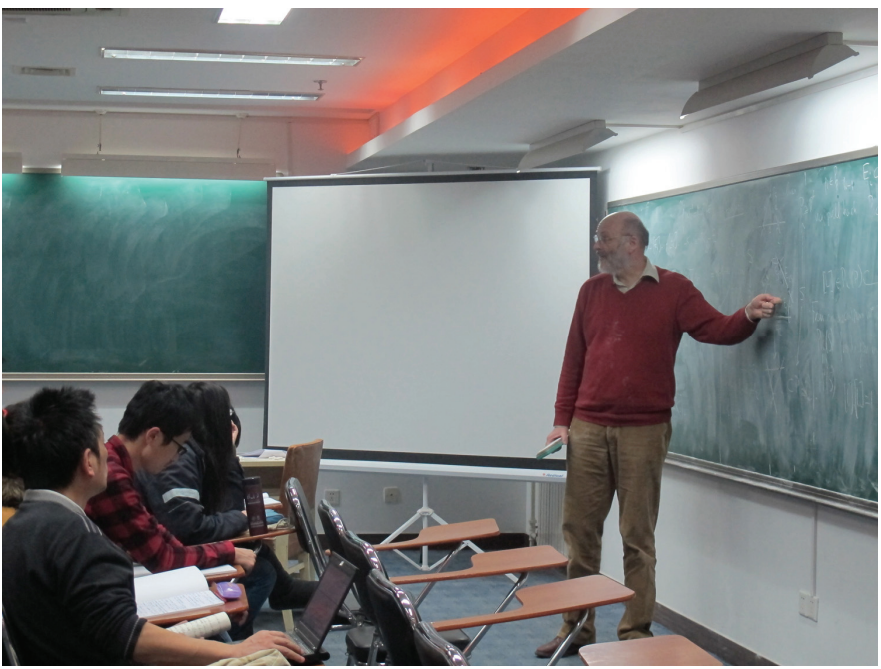

Eduard Looijenga

Parallel to an effort of recruiting full-time or permanent members, the Center also develops a visiting professor program. To ensure that these visiting professors will devote their energy in nurturing the junior members of our community, the Center requires every visiting professor to be in residence for at least one-month, and will deliver lecture series or seminar series as intensive as a regular course in Tsinghua. In summer 2010, junior participants in the MSC enjoyed the month-long lectures by S. Sternberg (Harvard) and J. Bost (Paris-Sud). By 2011, the MSC enjoyed the lectures by A. Beauville (Nice), D. Christodoulou (ETH), D. Gabai (Princeton), V. Kac (MIT), R. Hamilton (Columbia), R. Schoen (Stanford), S. Bloch (Chicago) and E. Looijenga (Utrecht). In 2012, Hamilton and Schoen returned to give three-monthlong lectures in the spring. Van der Geer (Utrecht) also visited for three-months in the fall. In 2010 alone, the MSC had twenty-two visiting professors who were in residence for at least one month. By 2011, the MSC received more than sixty visiting professors. In 2012, there were about seventy visiting professors. While the main audience of these visiting professors' courses was graduates and senior undergraduates of Tsinghua University, the courses also attracted excellent students from universities and institutes in Beijing and elsewhere, including a significant

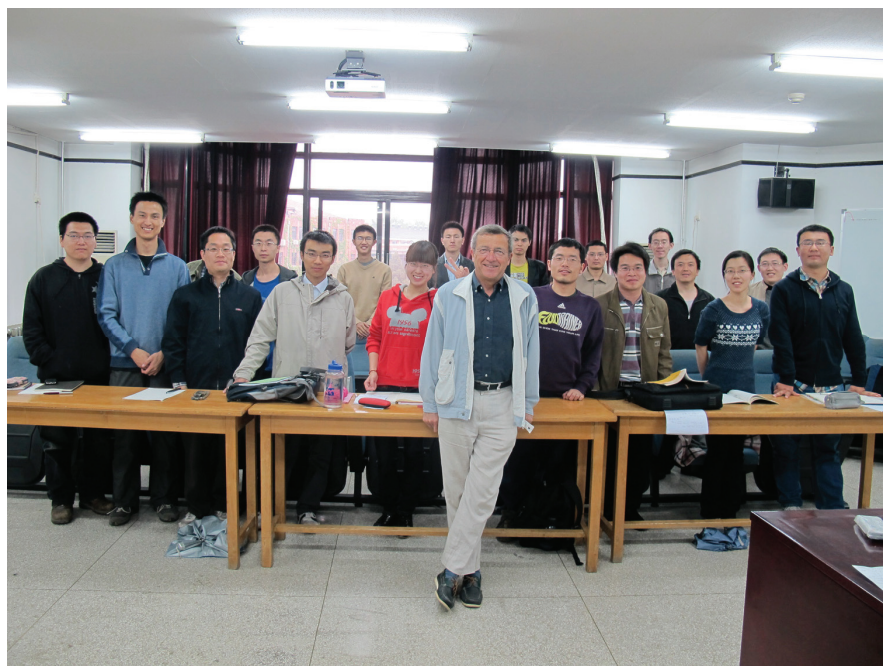

Arnaud Beauville

number of foreign students.

Some of these visitors had now turned into the MSC's permanent and full-time members, e.g. Looijenga. Some of them committed to return to MSC regularly to teach, e.g. Bloch, Van der Geer and Hamilton. By now, some of them have already made multiple visits and will continue to return, e.g. Christodoulou and Schoen.

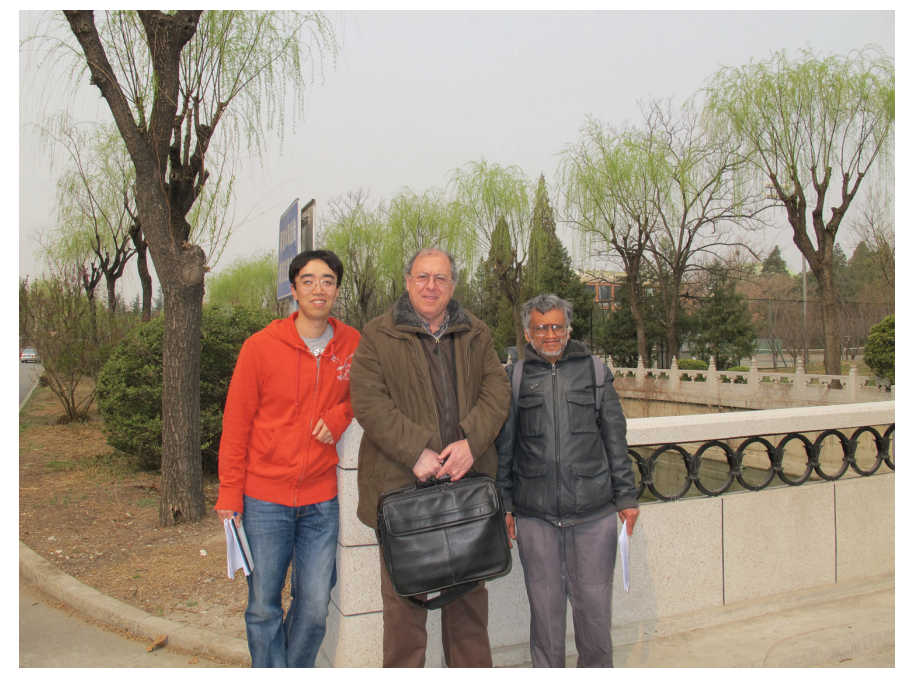

Left to right: Pin Yu (MSC, Tsinghua University), Demetrios Christodoulou (Eidgenössische Technische Hochschule Zürich), and Abul Masood-ul-Alam (MSC, Tsinghua University)

Such strong presence of senior international leading mathematicians is complemented by a crop of young and energetic talents and leading Chinese mathematicians. 
For example, Professors Jian-Guo Liu (Duke), Huai-Dong Cao (Lehigh), Feng Luo (Rutgers), Lizhen Ji (Michigan), Tonghai Yang (Wisconsin), David Gu (Stony Brook) and Yi Hu (Arizona) are committed to be in residence each year, and they will deliver instructional programs and advise junior members while in residence. In particular, Tonghai

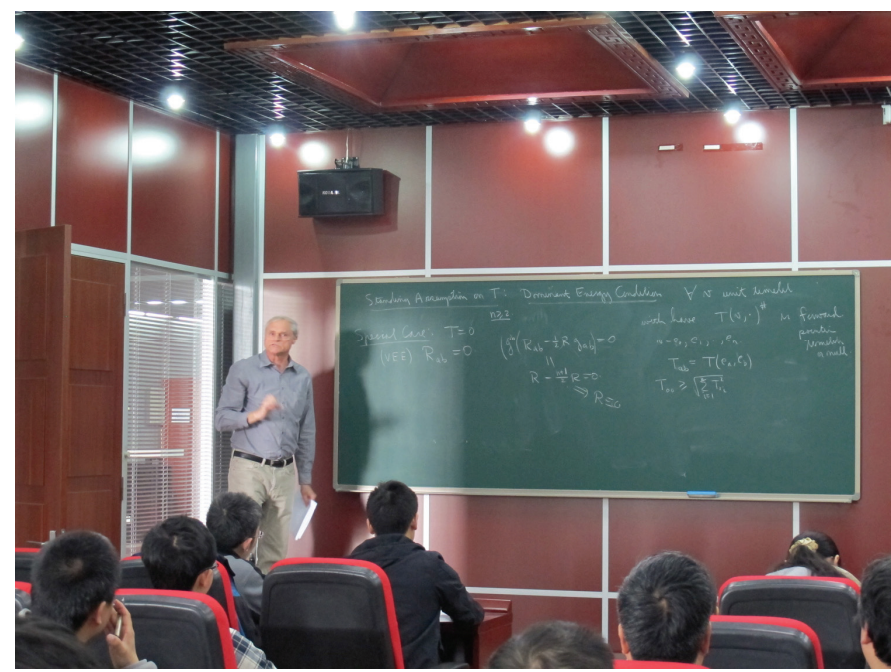

Richard Schoen, lecturing in the course of Mathematical Problems in General Relativity, Mar. 28-30, 2012

Yang also becomes a center member beginning in 2012, through the funding of 1000-Talents Visitor program of the Chinese Department of Education. As a capstone for

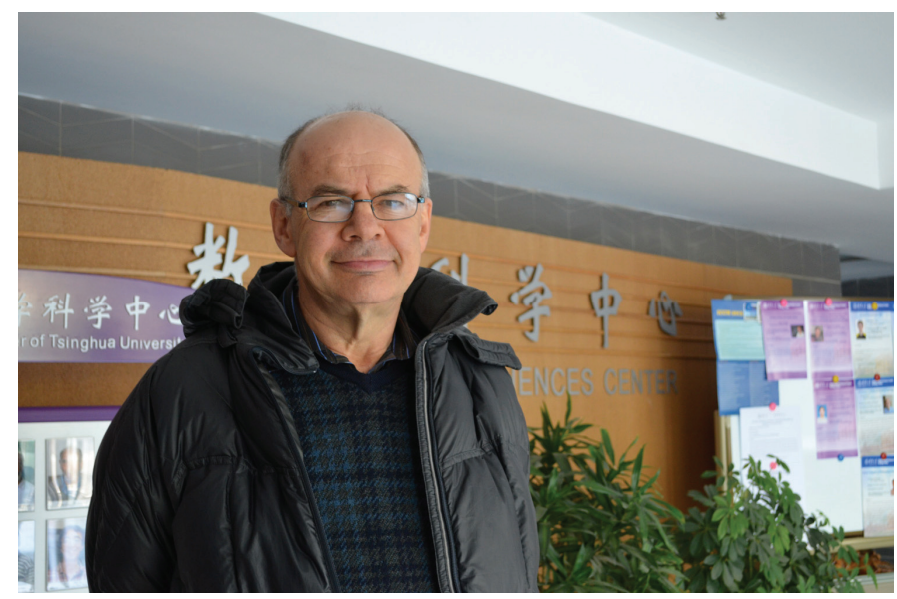

Hyam Rubinstein

the visiting professor program, in 2011 the MSC launched four Distinguished Lecture series. They are named after four mathematicians of Tsinghua University: Shiing-Shen Chern, Loo-Keng Hua, Pao-Lu Hsu and Chia-Chiao Lin. Each program invites up to two outstanding mathematicians each year to present multiple lectures on frontier topics.

The Chern Distinguished Lecture is established to honor Chern's monumental influence to geometry and topology. Its selection committee is co-chaired by Jun $\mathrm{Li}$ and Kefeng Liu. Its membership includes Richard Schoen, Demetrios Christodoulou, Peter Li, Shiu-Yuen Cheng, Fedor Bogomolov, Chris Hacon, Eduard Looijenga, and Richard Thomas. The past Chern Lecturers were Richard Schoen (Stanford), Eduard Looijenga (Utrecht), Clifford Taubes (Harvard) and Vaughan F. R. Jones (Vanderbilt).

The Hua Distinguished Lecture is established to honor Hua's fundamental contribution in number theory, algebra and analysis. Its selection committee is co-chaired by Horng-Tze Yau and Shouwu Zhang. Its committee membership includes John Coates, Jean-Marc Fontaine, Benedict Gross, Luis Caffarelli, Herbert Spohn, and Wilhelm Schlag. The past Hua Lecturers were Cedric Villani (Poincare Institute), Wilfried Schmid (Harvard), Luis A. Caffarelli (Austin), and jointly Shouwu Zhang (Princeton) and Ye Tian (Morningside Center).

The Hsu Distinguished Lecture is established to honor Hsu's instrumental contributions to the development of statistics and probability, especially in China. Its selection committee is chaired by Tze Leung Lai. Its Ex-

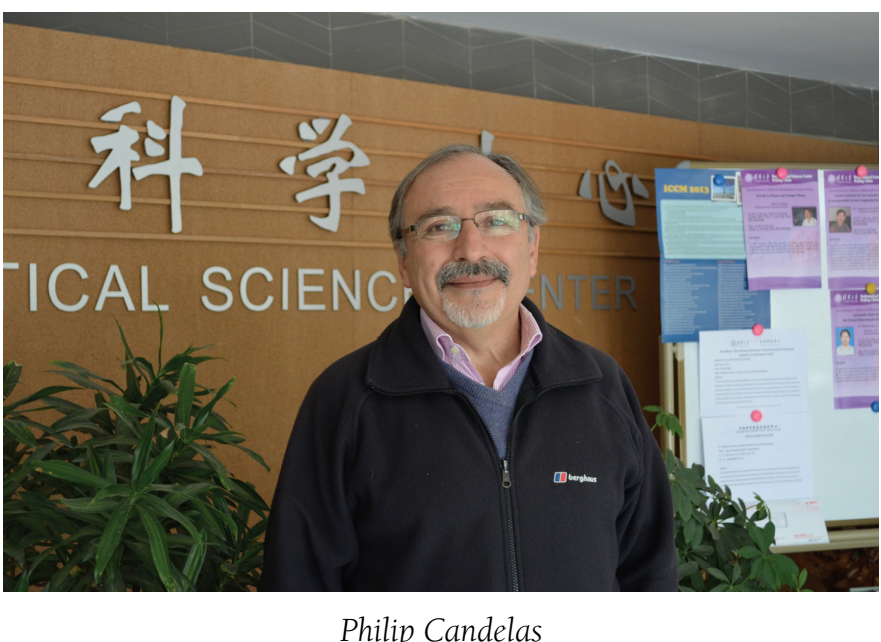

ecutive Chair is Xihong Lin. Its committee membership includes Jun Liu, Jianqing Fan, Wing H Wong, Sam Kou, and Heping Zhang. Its past lecturers were Bradley Efron (Stanford), Horng-Tzer Yau (Harvard), and Donald B. Rubin (Harvard).

The Lin Distinguished Lecture is established to honor Lin's pioneering contribution in applied mathematics. Its selection committee is co-chaired by Chi-Wang Shu and Zhouping Xin. Committee membership includes Tony Chan, Bjorn Enquist, Tom Hou, Tao Luo, and Sijue Wu. The past Lin Lecturers were Stanley Osher (UCLA) and George C. Papanicolaou (Stanford).

We are glad to announce that S. R. Srinivasa Varadhan (New York University) and Professor Peter Bickel (University of California, Berkeley) will give Pao-Lu Hsu Distinguished Lecture in 2013, Professor Michael Berry (University of Bristol) will give Chia-Chiao Lin Distinguished Lecture in the coming semester. 


\section{Research Programs}

With an increasing number of full-time members and

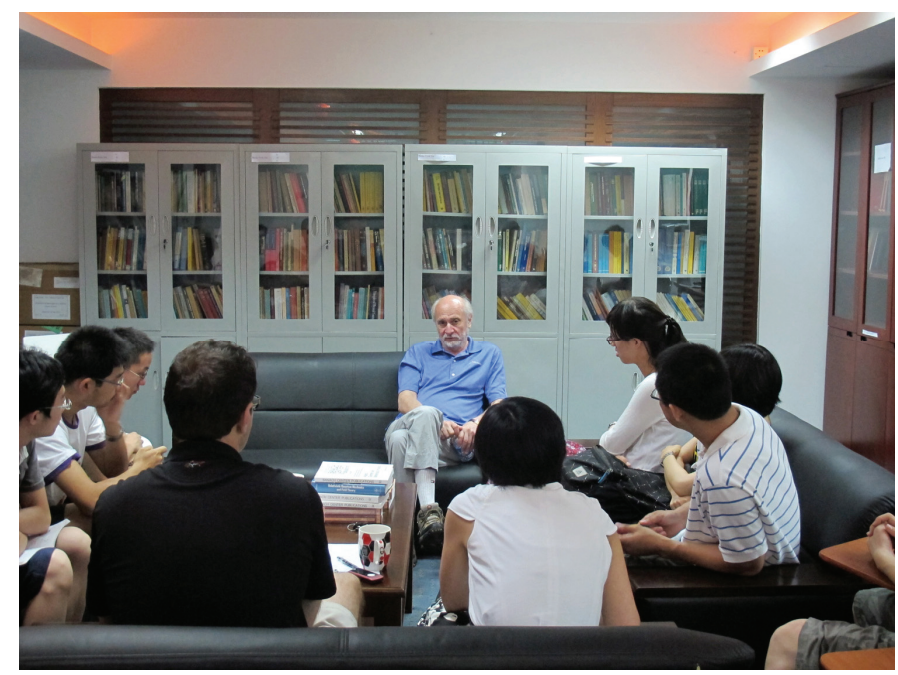

Donald B. Rubin

permanent members, in 2010 the MSC began a process of recruiting junior members to its rank, including post-doctorate fellows and graduate students. The first cohort of post-doctorate fellows joined the MSC in summer 2011. The first cohort of graduate students arrived at the MSC in summer 2012. By fall 2013, the MSC will have at least eight post-doctorate fellows and ten graduate students. The composition of the junior members of the MSC is just as internationalized as its faculty members. While most of them are Chinese, three of them come from

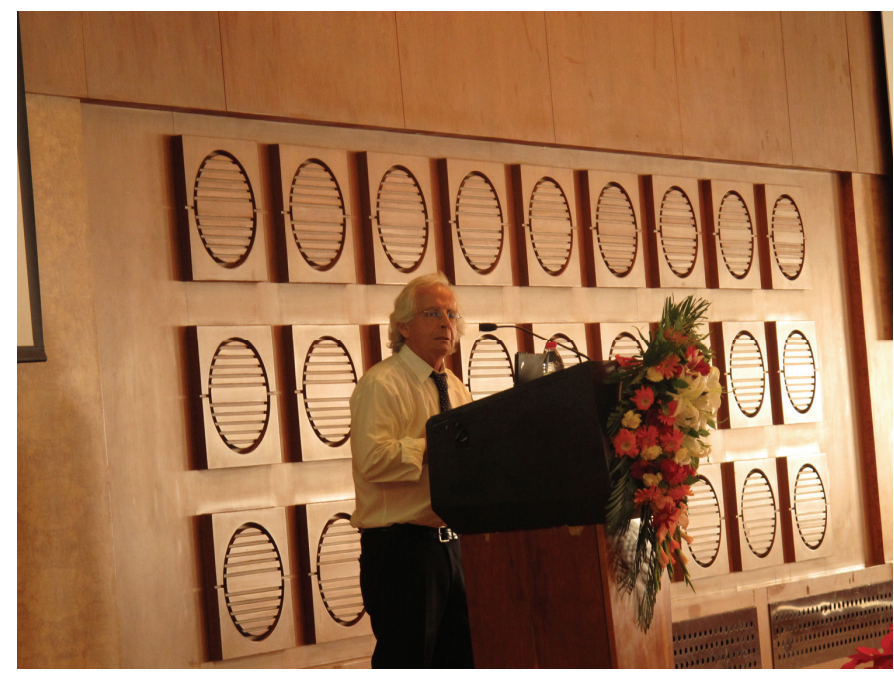

Stanley Osher

three different countries. With the synergy of the MSC's full-time members and its visiting professors, the MSC is now well positioned to further develop its academic program to train MSC students and junior fellows. After all, as Yau noted in various occasions, the success of a center is greatly reflected by how well it recruits and nurtures its junior fellows; namely its graduate students, post-doctorate fellows and tenure track assistant professors. In other words, the success of these junior fellows is the ultimate challenge to the MSC. It is also the driving force behind MSC's recruitment and visiting professor program. At presence, the Center is developing five programs. These programs are the collaborative results of the Center's members, long-term visiting scholars, the Center's scientific advisors, international advisors and faculty members of the Mathematical Sciences Department of Tsinghua University.

- Geometric Analysis. This program includes differential geometry, geometric flow and general relativity. S. T. Yau leads this program personally.

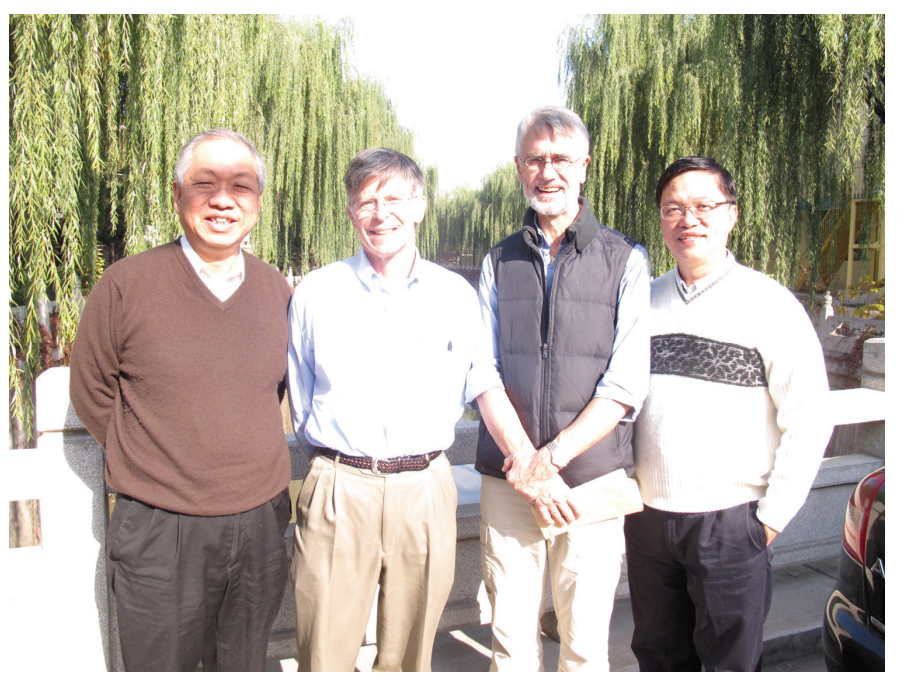

Left to right: Shing-Tung Yau, Eric Bedford (Indiana University), Gert Heckman (Radboud University in Nijmegen), Yat Sun Poon (University of California, Riverside) in Tsinghua campus. Photo taken during the 2011 fall semester.

- Algebra and Algebraic Geometry. This program enjoys the full-time and permanent residence of Looijenga, the part-time residence of Bloch and the presence of several junior members.

- Applied Analysis. This program has strong support from full-time senior member of the MSC (e.g. Tao Luo), members of the Department of Mathematical Sciences of Tsinghua and their close collaborators.

- Imaging. This program is enabled by a wide spectrum of expertise, from geometric topology, numerical analysis, computational mathematics, software designs to hardware implementation. S. T. Yau leads this program personally. 
- Mathematical Physics. At present, this program focuses on general relativity. Given the existing recruitment drive, this program will expand very quickly and very forcefully to include string theory in 2013.

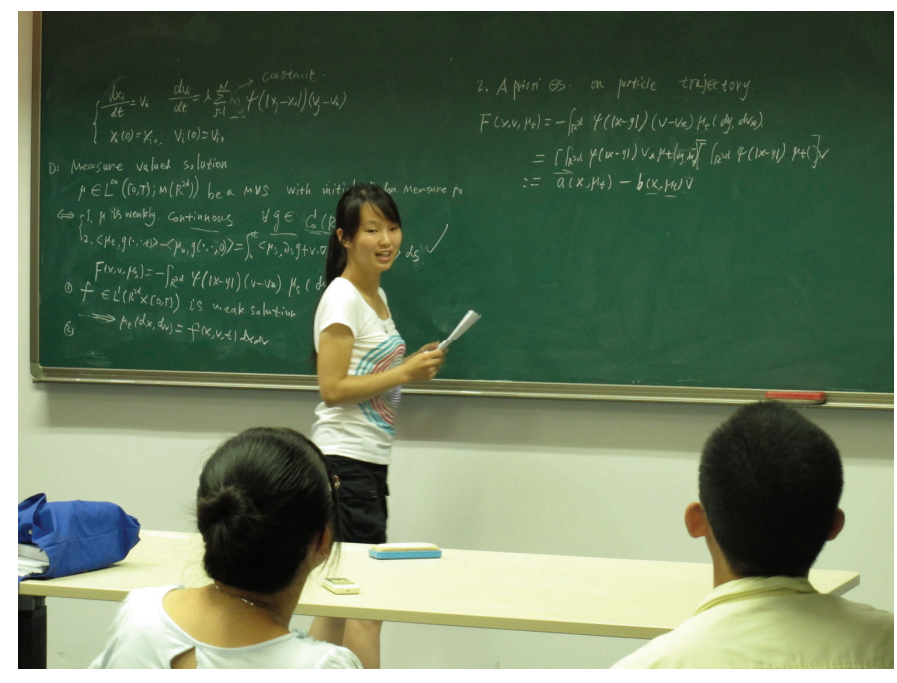

Students in the summer program 2011

- Statistics. Although this program at present has only one senior member, the annual part-time commitment from Jun Liu (Harvard) and the current recruitment success will gradually increase the strength of this program.

\section{International Conferences and Consortium of Applied Mathematicians}

Knowing that talents take energy to cultivate and to nurture, the MSC actively participates in the activities of a vertical infra-structure built over the years by S. T. Yau, namely the Morningside Prize, the New World Thesis Awards, the Yau College Mathematics Competition and the Yau High School Mathematics Awards. In 2010, in collaboration with several partner institutes, the MSC managed the ICCM 2010 and the ceremony of all these events. (See "Morningside Medals, the Chern Prizes, and the International Cooperation Awards" on page 113, and "The New World Mathematics Awards" on page 123.) Beginning in the same year, the MSC runs the New World Thesis Awards program annually. It is expected that the ceremony for the New World Mathematics Awards ceremony in 2013 will take place in the evening of July 14, on the first day of ICCM 2013.

The MSC co-hosts the annual S. T. Yau High School Mathematics Awards program with the Center of Mathematical Sciences of Zhejiang University. With donors' support, the MSC anticipates that this program will expand significantly in the next five years. The MSC also co-hosts the annual ceremony of Yau College Student Mathematics Contests.

In addition, the MSC hosts many professional conferences. In 2011, it co-hosted the "International Conference on Applied Mathematics and Statistics" with Morningside Center of Chinese Academy of Sciences and Information School of Renmin University in China. In 2012, it co-hosted the "International Conference on Complex Geometry, Singularities and Related Fields" with the Department of Mathematical Sciences of Tsinghua University.

Soon after the ICCM 2010, it became ripe to formalize the ICCM into a consortium of mathematicians and institutes. It started with applied mathematics. A group of applied mathematicians initiated the Consortium of Applied Mathematicians (CAM) in June 2012. It operates under ICCM.

The CAM-ICCM initiators are as follows: Raymond Chan (The Chinese University of Hong Kong), Hanfu Chen (Chinese Academy of Sciences), Xiaqi Ding (Chinese Academy of Sciences), Boling Guo (Institute of Applied Physics and Computational Mathematics), Thomas Yizhao Hou (California Institute of Technology), Tze Leung Lai (Stanford University), Chang-Shou Lin (National Taiwan University), Song-Sun Lin (National Chiao Tung University), Qun Lin (Chinese Academy of Sciences), Chi-Wang Shu (Brown University), Zhongci Shi (Chinese Academy of Sciences), Zikun Wang (Chinese Academy of Sciences), Zhouping Xin (The Chinese University of Hong Kong),

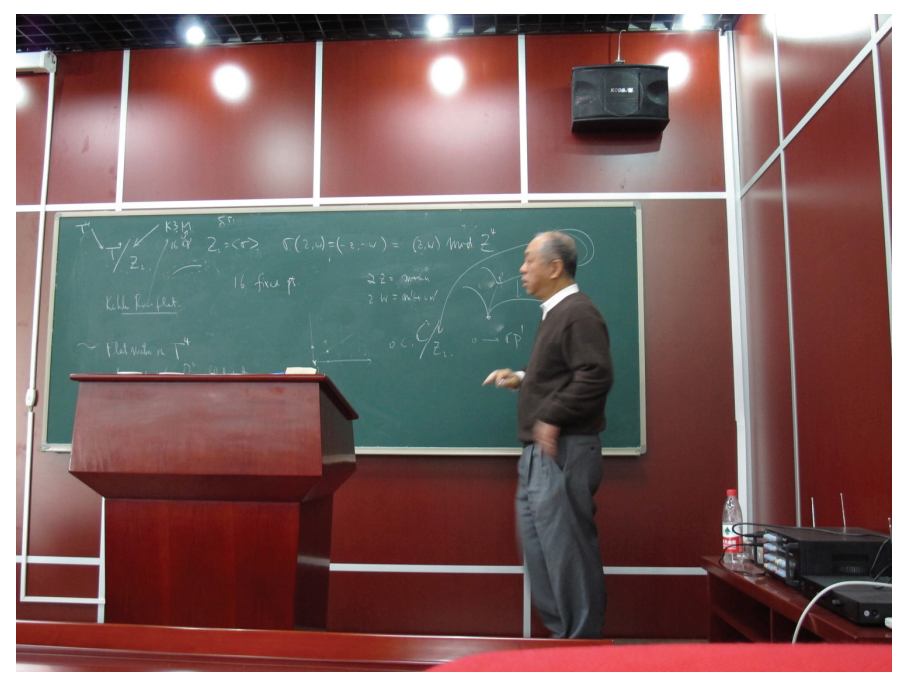

Shing-Tung Yau in the Fall program, 2011

Horng-Tzer Yau (Harvard University), Shing-Tung Yau (Harvard University) and Andrew Chi-Chih Yao (Tsinghua University).

This consortium is interested in the development of applied mathematics in Greater China area. Its goals are to identify opportunity for collaboration and promoting application of mathematics. This organization operates under ICCM. Its first chair is Professor Chi-Wang Shu of 
Brown University. The collaborative membership in Mainland China includes institutes in the Chinese Academy of Sciences, Tsinghua, Fudan, Zhejiang University, University of Sciences and Technology, Zhongshan, and Nanjiang. In Taiwan and Hong Kong, the membership includes institutes from Academic Sinica, Taiwan University, and in Chinese University of Hong Kong.

The inauguration ceremony of the Consortium of Applied Mathematicians, ICCM was held at Tsinghua University on June 29th, 2012. It was witnessed by officials from Chinese State Council, Ministry of Education, many dignitaries, students and professors.

It was subsequently decided that the CAM-ICCM's first academic activity was to host a workshop on "Imaging Science" on December 15-19, 2012, in Beijing. The first CAM-ICCM chose imaging science as its main theme to demonstrate the power of mathematics in applications and to motivate workshop participants to develop chal-

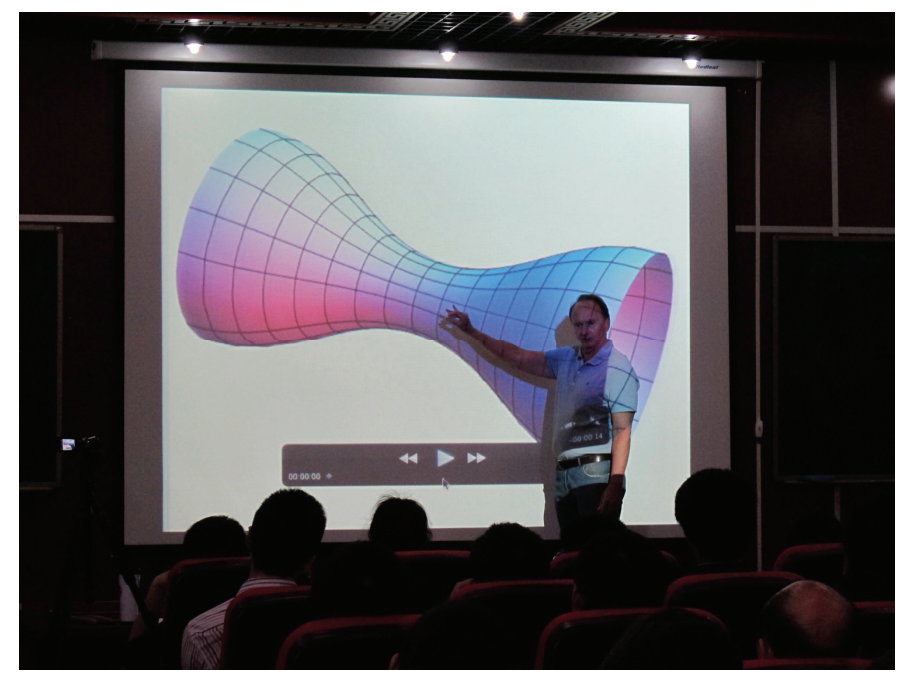

Richard Hamilton was giving a course on the Ricci Flow and the Poincaré Conjecture in June 2012

lenging mathematical and computational issues in imaging science.

This workshop was co-sponsored by CAM-ICCM, the Mathematical Sciences Center of Tsinghua University, and Institute for Pure \& Applied Mathematics of University of California, Los Angeles. This imaging workshop invited twenty-seven famous mathematicians to present their works.

Taking the opportunity of this workshop, CAM-ICCM celebrated Professor Stanley Osher's 70th birthday. Professor Stanley Osher is a pioneer in developing fundamental mathematics techniques such as level set methods, PDE based image processing, and optimization tools to advance imaging science. He has also contributed significantly to applied mathematics in China, through the training of graduate students and other young scholars and giving lectures in universities and research institutions in China.

\section{Tsinghua Sanya International Mathematics Forum}

To advance mathematics research worldwide and to elevate the research and academic level of the young scholars, the MSC expects broad and in depth international exchange. To fulfill a big part of this strategic goal, S. T. Yau initiated the first international mathematics conference center in China. His initiative receives strong support from Tsinghua University, Hainan Provincial Government, and Sanya City Government. Under the oversight of the MSC, in 2010 Tsinghua Sanya International Mathematics Forum (TSIMF) was established in Sanya.

The TSIMF's facilities will be built on a 140-acre land at Phoenix Hill of Phoenix Township; the land is surrounded by pristine environment and is neighboring to a golf course of a 5 -star resort facility. The total square footage of the facilities is over 28,000 square meter that includes state-of the art conference facilities, a large library, a hotel with 200 large guest rooms and the associated catering facilities, an Olympic size swimming pool, two tennis courts and other recreational facilities. The design of this facility is based on the concept that it will hold two international workshops simultaneously. Each could host 50-to-100 persons. These workshops in general are expected to last for five weekdays, and allow the weekends for transitions. In addition, there are family quarters to accommodate teams of researchers to be in residence to collaborate for substantial period of time.

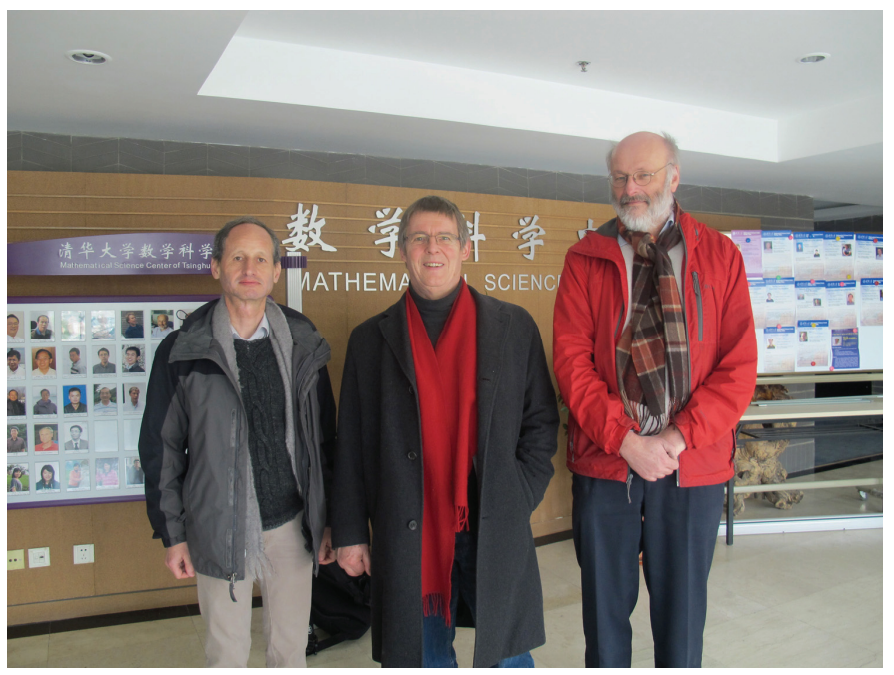

2013 MSC visitors from left to right: Michel Brion, Gert-Martin Greuel, and Eduard Looijenga

The construction of this facility will be completed in summer 2013.

Prior to its completion, the MSC had already run three conferences since the conception of the TSIMP. Its inaugural to third conference of TSIMF was held in December 2010, 2011 and January 2013 respectively. In December 2010, the speakers included Robbert Dijkgraaf (Amster- 
dam), Bjorn Engquist (Austin), Jean-Marc Fontaine (Paris), Benedict Gross (Harvard), David J. Gross (Santa Barbara) Richard Hamilton (Columbia), and Vaughan F. R. Jones (Vanderbilt). In December 2011, the TSIMF ran five parallel workshops. The principal speakers included Eduard Looijenga (Utrecht and Tsinghua), Shing-Tung Yau (Harvard), Jill Mesirov (MIT and Harvard), and Per Mykland (Chicago). In January 2013, the TSIMF ran four parallel workshops. The principal speakers included David Mumford (Brown), Richard Schoen (Stanford), Stanislav Smirnov (Geneva), and Wilfried Schmid (Harvard).

In December 2013, the TSIMF will host a grand

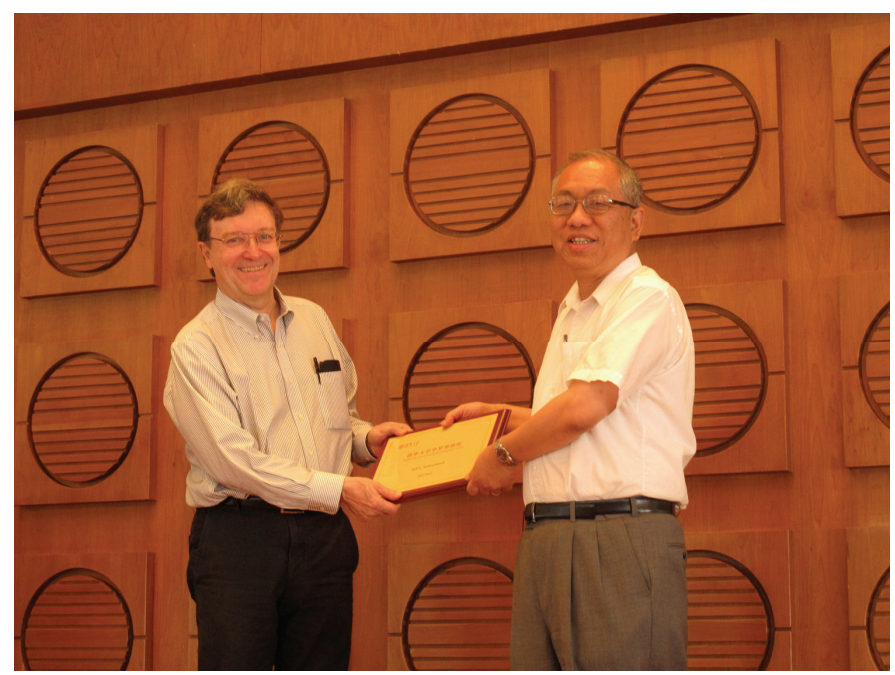

Left to right: Wilfried Schmid and Shing-Tung Yau

opening conference to officially unveil its new facility. We expect this conference will fill TSIMF facility to its capacity. At present the confirmed principal speakers include Yakov Sinai (Princeton), Ngô Bảo Châu (Chicago), and Clifford Taubes (Harvard).

Beginning in 2014, the TSIMF will begin to run weekly workshops. For each workshop, we expect about twenty speakers, with half of them from the U.S. and Europe, and half from Greater China area. The general participants are students or researchers from China and MSC's partner institutes. Travel expenses for participants from its partner institutes are expected to be paid by their home institutes while TSIMF will use its local fund source to provide room and board. At its full capacity, the TSIMF could accommodate one hundred weekly workshops annually.

\section{A supportive community behind MSC}

After three years, it is apparent that the Center is taking root. We are particularly encouraged by results of collaboration between center members and members of the math department in Tsinghua. The synergy and interaction among mathematicians in our community are extremely encouraging. We are also delighted to see its junior fellows getting promotion and recognition.
The development and the success of the Mathematical Sciences Center are no doubt the result of the leadership of the Tsinghua University administration and its director's vision and energy. It is also the result of a very strong international support. The Mathematical Sciences Center is advised by two committees; namely the International Advisory Committee and Scientific Committee. These committees support the Center in several ways. Most committee members personally participate in the Center's programs. Many assist the Center to compose and develop its academic and outreach programs. Some nominate participants. Others assist the Center to identify the rising talents for the Center to recruit. The membership of these two committees is given in the appendices.

On an institutional level, the MSC not only enjoys strong partnership with the Department of Mathematical Sciences in Tsinghua, but also strong support from many institutes and departments around the globe. It has now

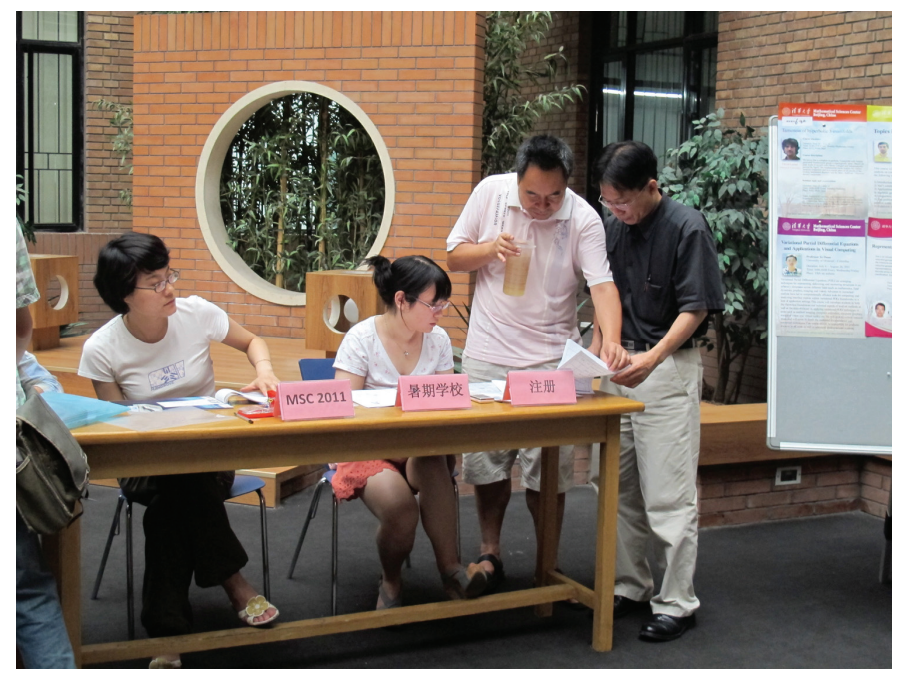

2011 Summer School Registration

established a network of institutional partners with the common goals of promoting mathematics and mathematics at their highest levels. With these institutional partners, listed in appendix, the MSC has already co-sponsored conferences, exchanged students and faculty visits. This network of international supports makes the vision of propelling the MSC to international forefront possible. It is amazing to this author that the list of MSC partners is growing as he writes.

On May 29, 2013, the MSC will host a ceremony to honor its existing as well as new institutional partners.

For the latest development of the MSC or this particular event, please visit its website at:

http://www.msc.tsinghua.edu.cn 


\section{Appendix A. International Advisory Committee 2012-2014}

1. Huai-Dong Cao, Lehigh University

2. Ching-Li Chai, University of Pennsylvania

3. John Coates, University of Cambridge

4. Benedict Gross, Harvard University

5. Stanislaw Janeczko, Stefan Banach Center, Polish Academy of Sciences

6. Lizhen Ji, University of Michigan

7. Ari Laptev, Institut Mittag-Leffler

8. Jill Mesirov, Massachusetts Institute of Technology

9. Stanley Osher, University of California, Los Angeles

10. Duong H. Phong, Columbia University

\section{Appendix B. Scientific Committee 2012-2014}

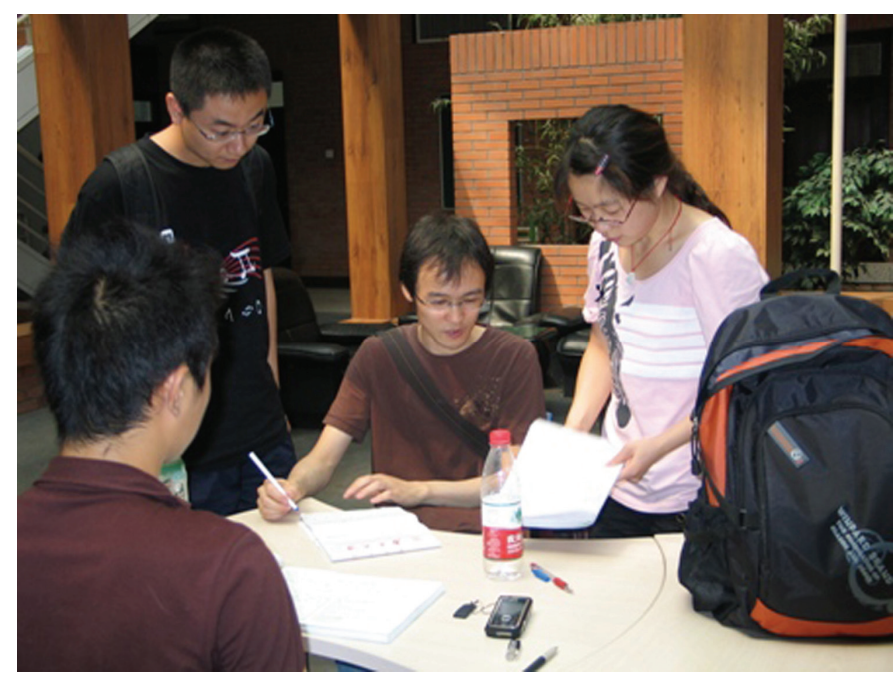

Students of the 2010 summer program

1. Chongqing Cheng, Nanjing University

2. Jiaxing Hong, Fudan University

3. Thomas Yizhao Hou, California Institute of Technology

4. Chang-Shou Lin, National Taiwan University

5. Jun Liu, Harvard University

6. Kefeng Liu, University of California, Los Angeles and Zhejiang University

7. Yat Sun Poon, University of California, Riverside

8. Xu-Jia Wang, Australian National University

9. Yuan Wang, Chinese Academy of Sciences

10. Jie Xiao, Tsinghua University

\section{Appendix C. Institutional Partners as of December 31, 2012}

- Morningside Center of Mathematics, Chinese Academy of Sciences

- Center of Mathematical Sciences, Zhejiang University
- Institute of Mathematical Sciences, the Chinese University of Hong Kong

- Center of Mathematical Modeling and Scientific Computing NCTU, Taiwan

- Taida Institute for Mathematical Sciences, Taiwan

- Institute for Pure and Applied Mathematics, University of California, Los Angeles

- The Stefan Banach International Mathematical Center, Institute of Mathematics, Polish Academy of Sciences, Poland

- The Mathematical Sciences Institute, Australian National University, Australia

- Institut Mittag-Leffler, the Royal Swedish Academy of Sciences, Sweden

- Department of Mathematical Sciences, Norwegian University of Science and Technology, Norway

- The Steklov Institute of Mathematics, Russian Academy of Sciences, Russia 